\title{
Impact of vitamin D level in diabetic people with peripheral neuropathy
}

\author{
Mohammad H. Assy' ${ }^{1}$ Nashwa A. Draz ${ }^{1}$, Sabah E. Fathy ${ }^{2^{*}}$ and Mohammad G. Hamed ${ }^{1}$
}

\begin{abstract}
Background: Diabetes mellitus (DM) is a metabolic disease which is complicated by occurrence of diabetic peripheral neuropathy (DPN). Vitamin D deficiency contributes to the etiology and progression of type 2 DM and development of micro-vascular complications so in this study we assessed vitamin D level in diabetic patients to evaluate the association between vitamin D level and occurrence of diabetic neuropathy and to assess if there is relationship with certain subtypes of diabetic neuropathy. This case-control study was conducted on 80 type 2 diabetic patients divided into four groups equally. (A): Diabetic patients with painful diabetic neuropathy. (B): Diabetic patients with painless diabetic neuropathy. (C): Diabetic patients with painless neuropathy, but have neuropathic ulcer. (D): Diabetic patients without neuropathy. All patients underwent clinical, neurological examination and nerve conduction study. Then CBC and vitamin D were estimated in the studied groups.

Results: Vitamin D level among the studied painful diabetic neuropathy group (A) ranged from 5.3 to $40.5 \mathrm{ng} / \mathrm{dl}$ with mean $17.4 \pm 10.9$. $70 \%$ of them had deficient vitamin D level. In the painless diabetic neuropathy group (B), vitamin D level ranged from 6.5 to $35.5 \mathrm{ng} / \mathrm{dl}$ with mean $18.9 \pm 8.49$. 60\% of them had deficient vitamin D level, while only $5 \%$ of the diabetic patients without neuropathy had deficient vitamin D level. There is significant negative correlation between vitamin D level and score of neuropathy where the lower vitamin D level the higher neuropathy score.
\end{abstract}

Conclusion: Lower vitamin D levels were found in diabetic patients with neuropathy especially those with painful neuropathy.

Keywords: DPN, Vitamin D, Neuropathic ulcer, DM type 2

\section{Background}

Diabetes mellitus, a world significant health concern, is a metabolic disease caused by defect in insulin secretion or an obstacle of insulin function or both. Approximately 415 million people are affected worldwide nowadays and it is expected by WHO to be 642 in 2040, which means that diabetes epidemic will continue [1]. DPN, a common complication of diabetes mellitus, is found in $50 \%$ patients living with diabetes mellitus. About $11 \%$ of patients with DPN have chronic, painful symptoms,

\footnotetext{
${ }^{*}$ Correspondence: Drsabah85@yahoo.com

${ }^{2}$ Department of Neurology, Faculty of Medicine, Zagazig University,

Zagazig, El-Sharkia, Egypt

Full list of author information is available at the end of the article
}

which seriously affect their lives, thus DPN is one of the main causes of morbidity and increased mortality [2].

The pathophysiology of DPN is still not well understood, and there are no universally accepted disease-modifying treatments for DPN and that is why the mainstay of treatment depends on symptoms control with pharmacotherapy that has limited efficacy and often their significant side effects limit their use [3]. Previous studies had identified the main risk factors associated with development of DPN including hyperglycemia itself, elevated glycated hemoglobin, and duration of the disease, elevated albumin excretion rates and obesity. However, the pathological progress of DPN is still not completely clear, so it is necessary to assess other potential risk factors that could be associated with development of DPN for better management [3]. Vitamin D is considered as a steroid 
hormone which has extensive effects in the human body. Vitamin D has already been proven to be associated with the regulation of bone metabolism and other metabolic processes, while recently it was found that vitamin D has strong association with some diseases as autoimmune disease [4].

Studies also have shown impaired insulin synthesis and secretion in animal models with vitamin $\mathrm{D}$ deficiency; diabetes onset can be delayed with 1-25-OH vitamin D intake, and some specific studies have reported that vitamin $\mathrm{D}$ deficiency contributes to the etiology and progression of type 2 diabetes and development of diabetes complications especially micro-vascular complications [5]. The relationship between vitamin D and DPN was suggested in several studies. In this study, we assessed vitamin D levels in diabetic patients with different types of DPN to evaluate the role of vitamin D as a potential risk factor which will lead to better management of the disease.

Therefore, this study aimed to evaluate the association between vitamin D level and diabetic neuropathy development, and to assess if there is a relationship with certain subtypes of diabetic neuropathy.

\section{Methods}

This case-control study was carried out in Endocrinology unit and outpatient clinic of Internal Medicine Department from July 2019 to February 2020, including 80 diabetic patients with type $2 \mathrm{DM}$ according to WHO criteria. The exclusion criteria included patients with non-diabetic neuropathies, alcoholics, patients with other systemic disorders like hepatic failure or renal failure and those on high-dose vitamin D supplementation were also excluded.

A total number of 80 diabetic patients were included in the study and were divided into four groups equally. Group A: included 20 diabetic patients (10 males and 10 females) who already developed painful diabetic neuropathy. Group B: included 20 diabetic patients (10 males and 10 females) who already developed painless diabetic neuropathy with no ulcers in leg. Group C: included 20 diabetic patients (9 males and 11 females) with painless neuropathy, but have neuropathic ulcer. Group D: included 20 diabetic patients (6 males and 14 females) without neuropathy (had no symptoms of neuropathy with normal nerve conduction study). All had seasonal sunlight exposure and doing activities, nearly all our patients live in sunny areas and the majority of them were collected during the summer months.

A detailed medical history was obtained from all patients, with special concern paid to the duration of diabetes, control of diabetes, their hypoglycemic medications and history of vitamin D supplements. General and neurological examination was done with assessment of the neuropathy severity using Toronto Clinical Scoring System (TCSS) and was interpreted as the following $0-5$ had no neuropathy, 6-8 had mild neuropathy, 9-11 had moderate neuropathy and $\geq 12$ had severe neuropathy [6].

Nerve conduction study was done for all patients including DPN with foot ulcerations using Nihon Kohden machine at neurophysiology unit of neurology department. Motor distal latency, amplitude and conduction velocity were assessed for both median, ulnar, common peroneal and posterior tibial nerves. Also, measurement of both sensory distal latency and amplitude of median, ulnar, sural and superficial peroneal nerves was done.

$5 \mathrm{ml}$ venous blood was taken from all patients under strict sterile conditions, centrifuged and the serum was used for assessment of 25-hydroxyvitamin D (the major circulating form of vitamin D and considered as the best indicator for vitamin D level) level using enzyme-linked immunosorbent assay (ELISA) method. The results were recorded as sufficient vitamin $D(\geq 30 \mathrm{ng} / \mathrm{dl})$, insufficient vitamin D (20-29 $\mathrm{ng} / \mathrm{dl})$ and deficient vitamin D $(<20 \mathrm{ng} / \mathrm{dl})$ [7].

This study was approved by our institutional review boards and informed consent was obtained from each patient before starting the study.

Statistical analysis was done using Statistical Package for Social Science (SPSS) version 22.0, 2013 created by IBM, Armonk, NY, USA. Quantitative data were expressed as mean \pm standard deviation (SD) and range, and the categorical variables were expressed as a number and percentage. Quantitative data were tested for normality using Kolmogorov-Smirnov test. Qualitative data were expressed as frequency and percentage. The percentages of categorical variables were compared using the Chi-square test, Fisher exact test was applied when the number of participants is less than 5 in the cell. $P$-value was considered to be statistically significant when $<0.05$.

\section{Results}

There was non significant difference among the patient groups regarding the demographic characteristics (Table 1). The present results showed that duration of diabetes is an important factor for the development of DPN as the mean of diabetes duration is obviously lower in diabetic patients without neuropathy $(3.6 \pm 1.2)$ when compared with other patient groups. Our patients were using different drug therapies for control of diabetes and the relation between the therapy used and the development of different types of neuropathy could not be assessed properly as most of our patients used different drug regimens during the disease course. The score of neuropathy among the studied painful diabetic 


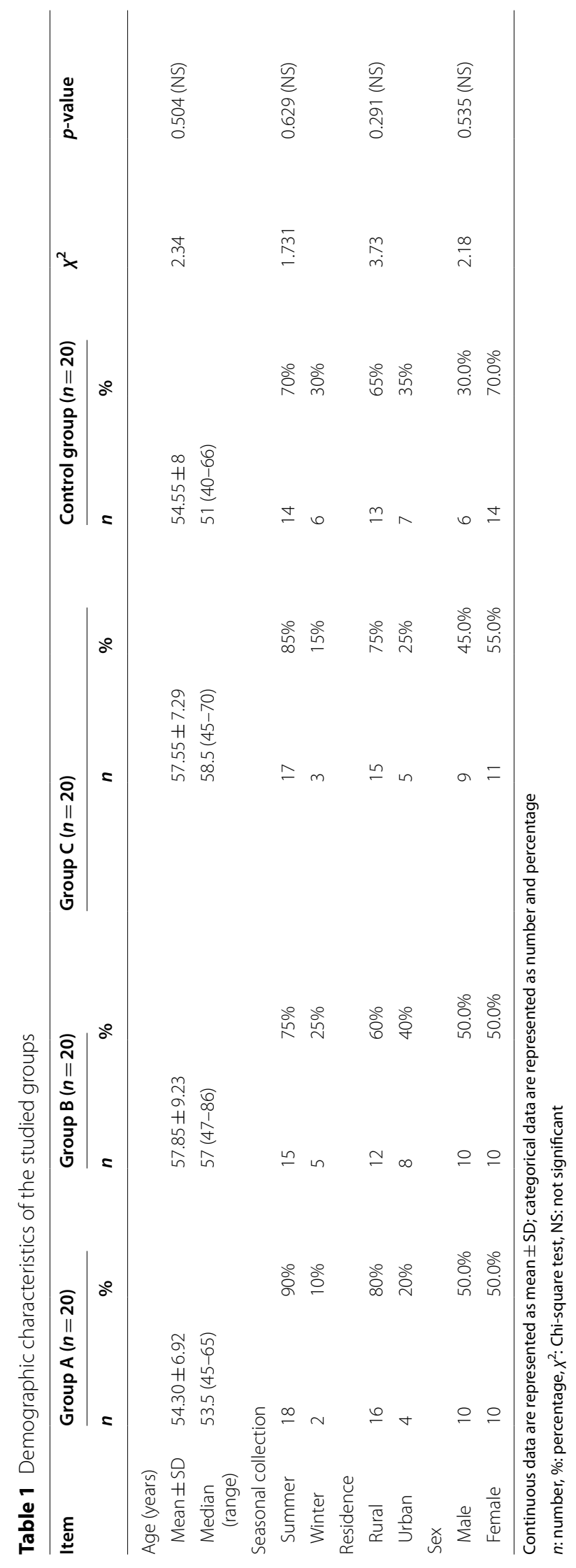


neuropathy group (group A) ranged from 9 to 15 with mean $12.25 \pm 2.14$. $40 \%$ of them had diabetic foot changes at different stages, while in the painless diabetic neuropathy group (group B) neuropathy score ranged from 6 to 9 with mean $7.6 \pm 1.09$, whereas in patients involved in group $C$ the neuropathy score ranged from 7 to 14 with mean $9.70 \pm 2.02 .6 \pm 1.09$. There was highly statistically significant difference between four groups regarding the scoring of neuropathy and staging of diabetic foot (Table 2).

With regard to the results of nerve conduction studies among the patients, we found that $100 \%$ of lower limb nerves are affected in patient groups, with sural and peroneal nerves being the most affected.

Regarding vitamin D level, the value of vitamin D among the studied painful diabetic neuropathy group (group A) ranged from 5.3 to $40.5 \mathrm{ng} / \mathrm{dl}$ with mean $17.4 \pm 10.9$. Seventy $\%$ of them had deficient vitamin D level. In the painless diabetic neuropathy group (group B) neuropathy, vitamin D level ranged from 6.5 to $35.5 \mathrm{ng} /$ $\mathrm{dl}$ with mean $18.9 \pm 8.49 .60 \%$ of them had deficient vitamin D, whereas only $5 \%$ of the diabetic patients without neuropathy had deficient vitamin D level. There was highly statistically significant difference between the four groups regarding vitamin D level and vitamin D status (Table 3).

There is a significant negative correlation between vitamin D level and score of neuropathy where the lower vitamin $\mathrm{D}$ level, the higher neuropathy score $(r=-0.325$, $p<0.05)$ as shown in Table 4.

The best fitting logistic regression model for vitamin D status was done. The table displays that age and score of neuropathy were the only statistically significant independent predictors of vitamin D status. A logistic regression was performed to determine the effects of sex, age, stage of diabetic foot, score of neuropathy, hemoglobin level, WBCs, platelets and albumin levels on the likelihood that participants deficient vitamin D. Patients with higher score of neuropathies were 1.3 times more likely to have vitamin $\mathrm{D}$ deficiency as shown in Table 5. Old age and higher score of neuropathy were proven to be associated with low vitamin $\mathrm{D}$ when compared with other factors in a logistic regression analysis (Table 6).

\section{Discussion}

DM and its related complications are increasing worldwide. DPN is considered a major micro-vascular complication, which is estimated to affect up to half of these diabetic patients and represent a main cause of mortality and morbidity in these patient. The complex pathogenesis of DPN is still not clear [8]. However, increased blood glucose level, decreased blood flow, hypoxia, hypoxia-induced pro-angiogenesis, and pro-inflammatory responses may play an important role in the pathogenesis. Moreover, pro-inflammatory cytokines, like

Table 2 Clinical characteristics of the studied patients

\begin{tabular}{|c|c|c|c|c|c|c|c|c|c|c|c|c|c|c|}
\hline \multirow[t]{2}{*}{ Item } & \multicolumn{3}{|c|}{ Group A $(n=20)$} & \multicolumn{3}{|c|}{ Group B $(n=20)$} & \multicolumn{3}{|c|}{ Group C $(n=20)$} & \multicolumn{3}{|c|}{$\begin{array}{l}\text { Control group } \\
(n=20)\end{array}$} & \multirow[t]{2}{*}{$x^{2} / \mathrm{KWT}$} & \multirow[t]{2}{*}{$p$-value } \\
\hline & $n$ & $\%$ & & $n$ & $\%$ & & $n$ & $\%$ & & $n$ & $\%$ & & & \\
\hline \multicolumn{15}{|l|}{ Duration of DM (years) } \\
\hline Mean $\pm S D$ & $8.95 \pm 3.42$ & & & $8.7 \pm 3.63$ & & & $7.6 \pm 2.94$ & & & $3.6 \pm 1.2$ & & & 14.86 & $0.001^{*}(\mathrm{HS})$ \\
\hline Median (range) & $9(4-15)$ & & & $8(3-17)$ & & & $7(3-14)$ & & & $3(1-6)$ & & & & \\
\hline \multicolumn{15}{|c|}{ The current hypoglycemic drug } \\
\hline Insulin & 10 & $50 \%$ & & 8 & $40 \%$ & & 8 & $40 \%$ & & 9 & $45 \%$ & & 4.81 & $0.850(\mathrm{NS})$ \\
\hline Glimepiride & 3 & $15 \%$ & & 5 & $25 \%$ & & 6 & $30 \%$ & & 5 & $25 \%$ & & & \\
\hline Gliclazide & 6 & $30 \%$ & & 3 & $15 \%$ & & 4 & $20 \%$ & & 3 & $15 \%$ & & & \\
\hline Combined preparation & 1 & $5 \%$ & & 4 & $20 \%$ & & 2 & $10 \%$ & & 3 & $15 \%$ & & & \\
\hline \multicolumn{15}{|l|}{ Score of neuropathy (TCSS) } \\
\hline Mean $\pm S D$ & $12.25 \pm 2.14$ & & & $7.6 \pm 1.09$ & & & $9.70 \pm 2.02$ & & & $0.95 \pm 1.19$ & & & 63.39 & $0.000^{*}(\mathrm{HS})$ \\
\hline Median (range) & $12.5(9-15)$ & & & $8(6-9)$ & & & $9(7-14)$ & & & $0.5(0-4)$ & & & & \\
\hline \multicolumn{15}{|l|}{ Stage of diabetic foot } \\
\hline Normal foot & 12 & & $60 \%$ & 20 & & $100 \%$ & 0 & & $0 \%$ & 20 & & 10 & 65.11 & $0.001^{*}(\mathrm{HS})$ \\
\hline Ulcerated & 4 & & $20 \%$ & 0 & & $0 \%$ & 17 & & $85 \%$ & 0 & & 0 & & \\
\hline Infected & 3 & & $15 \%$ & 0 & & $0 \%$ & 2 & & $10 \%$ & 0 & & 0 & & \\
\hline Necrotic & 1 & & $5 \%$ & 0 & & $0 \%$ & 1 & & $5 \%$ & 0 & & 0 & & \\
\hline
\end{tabular}

$n$ : number; \%: percentage; $x^{2}$ : Chi-square test; KWt: Kruskal-Wallis test; HS: highly significant; TCSS: Toronto Clinical Scoring System 
Table 3 Nerve conduction study of patients

\begin{tabular}{|c|c|c|c|c|c|c|c|c|}
\hline \multirow[t]{2}{*}{ Item } & \multicolumn{2}{|c|}{ Group A $(n=20)$} & \multicolumn{2}{|c|}{ Group B $(n=20)$} & \multicolumn{2}{|c|}{ Group C $(n=20)$} & \multicolumn{2}{|c|}{ Group D $(n=20)$} \\
\hline & $n$ & $\%$ & $n$ & $\%$ & $n$ & $\%$ & $n$ & $\%$ \\
\hline \multicolumn{9}{|l|}{ Lower limb nerves } \\
\hline Absent sural SNAP & 20 & 100 & 20 & 100 & 20 & 100 & 0 & 0 \\
\hline \multicolumn{9}{|l|}{ Peroneal CMAP } \\
\hline Normal & 12 & 60 & 0 & 0 & 0 & 0 & 20 & 100 \\
\hline Reduced & 7 & 35 & 10 & 50 & 5 & 25 & 0 & \\
\hline Absent & 1 & 5 & 10 & 50 & 15 & 75 & 0 & \\
\hline \multicolumn{9}{|l|}{ Tibial CMAP } \\
\hline Normal & 16 & 80 & 5 & 25 & 3 & 15 & 20 & 100 \\
\hline Reduced & 4 & 20 & 11 & 55 & 8 & 40 & 0 & \\
\hline Absent & 0 & 0 & 4 & 20 & 9 & 45 & 0 & \\
\hline \multicolumn{9}{|l|}{ Upper limb nerves } \\
\hline \multicolumn{9}{|l|}{ Median CMAP } \\
\hline Normal & 17 & 85 & 13 & 65 & 11 & 55 & 20 & 100 \\
\hline Reduced & 3 & 15 & 7 & 35 & 9 & 45 & & \\
\hline \multicolumn{9}{|l|}{ Ulnar CMAP } \\
\hline Normal & 19 & 95 & 18 & 90 & 15 & 75 & 20 & 100 \\
\hline Reduced & 1 & 5 & 2 & 10 & 5 & 25 & & \\
\hline Affected median SNAP & 3 & 15 & 4 & 20 & 4 & 20 & 1 & 5 \\
\hline Affected ulnar SNAP & 1 & 5 & 3 & 15 & 3 & 15 & 0 & 100 \\
\hline
\end{tabular}

SNAP: sensory nerve action potential; CMAP: compound motor action potential

Table 4 Vitamin D level among the studied groups

\begin{tabular}{|c|c|c|c|c|c|c|c|c|c|c|c|}
\hline \multirow[t]{2}{*}{ Item } & \multicolumn{2}{|c|}{ Group A $(n=20)$} & \multicolumn{2}{|c|}{ Group B $(n=20)$} & \multicolumn{2}{|c|}{ Group C $(n=20)$} & \multicolumn{3}{|c|}{ Control group $(n=20)$} & \multirow[t]{2}{*}{$x^{2} / \mathrm{KWt}$} & \multirow[t]{2}{*}{$p$-value } \\
\hline & $n$ & $\%$ & $n$ & $\%$ & $n$ & $\%$ & & $n$ & $\%$ & & \\
\hline \multicolumn{12}{|l|}{ Vitamin D level } \\
\hline Mean \pm SD & $17.4 \pm 10.9$ & & $18.9 \pm 8.49$ & & $20.12 \pm 9.55$ & & $32.1 \pm 7.9^{*}$ & & & 63.39 & $0.000^{*}(\mathrm{HS})$ \\
\hline Median (range) & $15.3(5.3-40.5)$ & & $17(6.5-35.5)$ & & $17.9(5.3-36.5)$ & & $31.2(16.3-53)$ & & & & \\
\hline \multicolumn{12}{|l|}{ Vitamin D status } \\
\hline Deficient (<20 ng/dl) & 14 & $70 \%$ & 12 & $60 \%$ & 13 & $65 \%$ & & 1 & $5 \%$ & 22.33 & $0.001^{*}(\mathrm{HS})$ \\
\hline Insufficient (20-30 ng/dl) & 3 & $15 \%$ & 3 & $15 \%$ & 3 & $15 \%$ & & 7 & $35 \%$ & & \\
\hline Sufficient ( $\geq 30$ ng/dl) & 3 & $15 \%$ & 5 & $25 \%$ & 4 & $20 \%$ & & 12 & $60 \%$ & & \\
\hline
\end{tabular}

$x^{2}$ : Chi-square test; KWt: Kruskal-Wallis test; $\mathrm{HS}$ : highly significant

Table 5 Correlation between vitamin D level and score of neuropathy

\begin{tabular}{ll}
\hline Correlation coefficient & Vit D level \\
\hline Score of neuropathy & \\
$R$ & $-0.325^{* *}$ \\
$p$-value & 0.003 \\
\hline${ }^{* *}$ Correlation is significant at the 0.01 level (2-tailed) &
\end{tabular}

interleukins, affect nerves and glial cells and are supposed to be involved in the pathology of diabetic neuropathy. Vitamin D deficiency is linked to the presence of inflammation and hyperglycemia, so it could be considered as a high risk factor for DPN [9].

The current study was conducted on 80 patients with type $2 \mathrm{DM}$ aiming to evaluate the association between vitamin D level and diabetic neuropathy development and to assess if there is a relationship with certain subtypes of diabetic neuropathy.

In the current study, we found that $100 \%$ of lower limb nerves are affected in patient groups and this affection is seen mostly in sensory nerves, which is in accordance with the rule that diabetic neuropathy 
Table 6 Logistic regression of vitamin D deficiency on presence of risk factors among the studied groups

\begin{tabular}{|c|c|c|c|c|c|c|c|}
\hline \multirow[t]{2}{*}{ Variables } & \multirow[t]{2}{*}{$B$} & \multirow[t]{2}{*}{ S.E } & \multirow[t]{2}{*}{ Wald } & \multirow[t]{2}{*}{$p$-value } & \multirow[t]{2}{*}{$\operatorname{Exp}(B)$} & \multicolumn{2}{|c|}{$95 \% \mathrm{Cl}$ for $\operatorname{EXP}(B)$} \\
\hline & & & & & & Lower & Upper \\
\hline Male sex & -0.447 & 0.785 & 0.324 & 0.569 & 0.640 & 0.137 & 2.977 \\
\hline Age & 0.100 & 0.050 & 4.000 & $0.045^{*}$ & 1.105 & 1.002 & 1.219 \\
\hline Ulcerated foot & 0.452 & 1.723 & 0.069 & 0.793 & 1.571 & 0.054 & 46.034 \\
\hline Infected foot & 0.657 & 1.577 & 0.173 & 0.677 & 1.928 & 0.088 & 42.392 \\
\hline Score of neuropathy & 0.330 & 0.120 & 7.500 & $0.006^{*}$ & 1.391 & 1.098 & 1.761 \\
\hline $\mathrm{Hb}$ & 0.061 & 0.469 & 0.017 & 0.897 & 1.063 & 0.424 & 2.666 \\
\hline WBCs & 0.028 & 0.166 & 0.028 & 0.867 & 1.028 & 0.742 & 1.424 \\
\hline Plt & -0.001 & 0.004 & 0.083 & 0.773 & 0.999 & 0.992 & 1.006 \\
\hline Albumin & 0.482 & 1.229 & 0.154 & 0.695 & 1.619 & 0.146 & 17.987 \\
\hline
\end{tabular}

$R^{2}=0.38$ Chi-square test for model coefficient $=24.4, p$-value $=0.011 *$ Variable(s) entered on equation: sex, age, stage of diabetic foot, score of neuropathy, hemoglobin, WBCs, platelets, albumin

affects mostly long sensory nerves earlier and this is in accordance with Vinik and colleagues [10].

We found that common peroneal nerve involvement predominates and this was supported by Kong and his colleagues [11], while Kakrani and colleagues [12] assumed that tibial nerve involvement is more likely to be found in DPN.

Our results are in agreement with a study of Shillo and his colleagues [13], who studied vitamin D level in patients with painful DPN, painless DPN, and diabetics without DPN and it showed that significant deficiency in patients with painful DPN more than patients with painless DPN and those without DPN.

In addition, this was in accordance with a previous study of Usluogullari and coauthors [14], who evaluated vitamin D level in 557 patients having type two diabetes and healthy controls randomly selected and revealed that vitamin $\mathrm{D}$ deficiency is more common in diabetic patients with micro-vascular complications including neuropathy. Vitamin D levels were found to be lower in patients in whom these complications were more severe.

In a study of Oraby and colleagues [15], who compared patients with DPN to healthy controls, vitamin D deficiency was found to be a suspected risk factor for DPN as the results of this study ensured that vitamin D levels in DPN patients were lower than those in the control group, especially in patients with severe neuropathy.

Also, Martin and colleagues [16] tried to assess the effectiveness of vitamin D supplementation on patients with DPN and found that vitamin D supplementation together with exercise reduced both symptoms and complications of DPN. As well, Papanas and Ziegler [17] analyzed risk factors for DPN in a major study and they concluded that vitamin D deficiency had a significant risk factor for development of DPN.
Moreover, $\mathrm{Qu}$ and his colleagues [18] directed a metaanalysis to evaluate the role of vitamin D deficiency in DPN over ten studies. They found that vitamin D level was significantly reduced in patients with DPN, but more in Caucasian than Asian races. This meta-analysis also assumed that it is clear that vitamin D is involved in the development of DPN, and vitamin D deficiency is very likely to be associated with increased risk of DPN. Appropriate vitamin D supplements also can be an effective tool to delay the development of DPN in diabetic patients.

Furthermore, another study was done by Greenhagen and colleagues [19] to evaluate vitamin D in patients with diabetic foot complications. In 100 patients involved in the study, it was found that $75 \%$ of patients had vitamin D deficiency, but no significant difference between patients with Charcot joints and those without. In another metaanalysis by Zhang and coauthors [20], involving more than 13 studies including about 2800 patients all with type two diabetes, heterogeneity test showed significant relation between vitamin D and development of DPN in T2DM patients. Most previous studies confirmed that there was vitamin D deficiency in diabetic patients with DPN. Also, more studies are needed to evaluate the definitive role of vitamin D deficiency in development of DPN.

There were no previous studies confirming the results against our study.

\section{Conclusion}

Our study concluded that, vitamin D deficiency plays an important role in the development of DPN mainly in the painful subtype, so we recommended further study to follow-up the improvement of painful neuropathic symptoms in those patients after vitamin D supplementation. Vitamin D could also aid in the improvement of the 
outcome in non-painful neuropathy. We recommended serial assessment of vitamin $\mathrm{D}$ levels in all diabetic patients as correction of vitamin D deficiency may delay the development of all subtypes DPN.

\section{Abbreviations}

DM: Diabetes mellitus; DPN: Diabetic peripheral neuropathy; T2DM: Type 2 diabetes mellitus; TCSS: Toronto Clinical Scoring System.

\section{Acknowledgements}

The authors would like to appreciate all participants and their families as well as the hospital staff who contributed to the study.

\section{Authors' contributions}

MHA designed the study and had done the statistical analysis. NAD was involved in literature search, data acquisition, and manuscript preparation. SEF was involved in manuscript preparation, analysis, and editing. MGH was involved in study design, analysis, and manuscript preparation. All authors have read and approved the final manuscript.

\section{Funding}

This study was not supported by any source of funding.

\section{Availability of data and materials}

Data and materials supporting the results of this article are included within the article.

\section{Declarations}

\section{Ethics approval and consent to participate}

The study was approved by the Institutional Ethics Committee of the Faculty of Medicine, Zagazig University (ZU-IRB \#5268/14-3-2019). Written informed consent was obtained from all study participants after explaining the details and benefits as well as risks to them. Surrogate consent from the patient's legal guardian or designated health proxy was permitted in cases where the patient did not have decision-making capacity.

\section{Consent for publication}

Not applicable.

\section{Competing interests}

The authors declared that they have no conflicts of interest with respect to the authorship and/or publication of this article.

\section{Author details}

${ }^{1}$ Department of Internal Medicine, Faculty of Medicine, Zagazig University, Zagazig, El-Sharkia, Egypt. ${ }^{2}$ Department of Neurology, Faculty of Medicine, Zagazig University, Zagazig, El-Sharkia, Egypt.

Received: 14 May 2021 Accepted: 16 August 2021

Published online: 31 August 2021

\section{References}

1. Ogurtsova K, da Rocha Fernandes JD, Huang Y, Linnenkamp U, Guariguata L, Cho NH, et al. IDF diabetes atlas: global estimates for the prevalence of diabetes for 2015 and 2040. Diabetes Res Clin Pract. 2017;128:40-50.
2. Tesfaye S, Selvarajah D. Advances in the epidemiology, pathogenesis and management of diabetic peripheral neuropathy. Diabetes Metab Res Rev. 2012;28(1):8-14.

3. Yagihashi S. Recent advances in clinical practice and in basic research on diabetic neuropathy. Brain Nerve. 2011;63(6):571-82.

4. Smith TJ, Lanham-New SA, Hart KH. Vitamin D in adolescents: are current recommendations enough? J Steroid Biochem Mol Biol. 2017;173:265-72.

5. Pittas AG, Lau J, Hu FB, Dawson-Hughes B. The role of vitamin D and calcium in type 2 diabetes. A systematic review and meta-analysis. J Clin Endocrinol Metab. 2007;92(6):2017-29.

6. Bril V, Perkins BA. Validation of the Toronto Clinical Scoring System for diabetic polyneuropathy. Diabetes Care. 2002;25(11):2048-52.

7. Ross AC, Manson JE, Abrams SA, Aloia JF, Brannon PM, Clinton SK, et al. The 2011 dietary reference intakes for calcium and vitamin D: what dietetics practitioners need to know? J Am Diet Assoc. 2011;111(4):524-7.

8. Cannell JJ, Grant WB, Holick MF. Vitamin D and inflammation. Dermatoendocrinology. 2015;6(1):e983401.

9. Bilir B, Tulubas F, Bilir BE, Atile N, Kara SP, Yildirim T, et al. The association of vitamin $D$ with inflammatory cytokines in diabetic peripheral neuropathy. J Phys Ther Sci. 2016;28(7):2159-63.

10. Vinik AI, Bril V, Litchy WJ, Price KL, Bastyr EJ, MBBQ Study Group. Sural sensory action potential identifies diabetic peripheral neuropathy responders to therapy. Muscle Nerve. (3rd). 2005;32(5):619-25.

11. Kong X, Lesser EA, Potts FA, Gozani SN. Utilization of nerve conduction studies for the diagnosis of polyneuropathy in patients with diabetes: a retrospective analysis of a large patient series. J Diabetes Sci Technol. 2008;2(2):268-74.

12. Kakrani AL, Gokhale VS, Vohra KV, Chaudhary N. Clinical and nerve conduction study correlation in patients of diabetic neuropathy. J Assoc Physicians India. 2014;62(1):24-7.

13. Shillo P, Selvarajah D, Greig M, Gandhi R, Rao G, Wilkinson ID, et al. Reduced vitamin D levels in painful diabetic neuropathy. Diabet Med. 2019;36(1):44-51.

14. Usluogullari CA, Balkan F, Caner S, Ucler R, Kaya C, Ersoy R, et al. The relationship between microvascular complications and vitamin D deficiency in type 2 diabetes mellitus. BMC Endocr Disord. 2015;25(15):33. https:// doi.org/10.1186/s12902-015-0029-y.

15. Oraby MI, Srie MA, Abdelshafy S, Elfar E. Diabetic peripheral neuropathy: the potential role of vitamin D deficiency. Egypt J Neurol Psychiatry Neurosurg. 2019;55:10.

16. Martin CL, Albers JW, Pop-Busui R, DCCT/EDIC Research Group. Neuropathy and related findings in the diabetes control and complications trial/epidemiology of diabetes interventions and complications study. Diabetes Care. 2014;37(1):31-8.

17. Papanas N, Ziegler D. Risk factors and comorbidities in diabetic neuropathy: an update 2015. Rev Diabet Stud. 2015;12(1-2):48-62.

18. Qu GB, Wang $L L$, Tang $X$, Wu W, Sun $Y H$. The association between vitamin $\mathrm{D}$ level and diabetic peripheral neuropathy in patients with type 2 diabetes mellitus: an update systematic review and meta-analysis. J Clin Transl Endocrinol. 2017;9:25-31.

19. Greenhagen RM, Frykberg RG, Wukich DK. Serum vitamin D and diabetic foot complications. Diabet Foot Ankle. 2019;10(1):1579631.

20. Zhang B, Zhao W, Tu J, Wang $X$, Hao Y, Wang H, et al. The relationship between serum 25-hydroxyvitamin D concentration and type 2 diabetic peripheral neuropathy: a systematic review and meta-analysis. Medicine (Baltimore). 2019;98(48):e18118.

\section{Publisher's Note}

Springer Nature remains neutral with regard to jurisdictional claims in published maps and institutional affiliations. 